\title{
Evidence for Quantization of Mechanical Rotation of Magnetic Nanoparticles
}

\author{
J. Tejada, ${ }^{1}$ R. D. Zysler, ${ }^{2}$ E. Molins, ${ }^{3}$ and E. M. Chudnovsky ${ }^{1,4}$ \\ ${ }^{1}$ Facultat de Física, Universitat de Barcelona, Diagonal 645, 08028 Barcelona, Spain \\ ${ }^{2}$ Centro Atómico Bariloche, 8400 S.C. de Bariloche, RN, Argentina \\ ${ }^{3}$ Institut de Ciència de Materials de Barcelona (C.S.I.C.), Campus de la Universitat Autònoma de Barcelona, 08193 Bellaterra, Spain \\ ${ }^{4}$ Physics Department, Lehman College, The City University of New York, \\ 250 Bedford Park Boulevard West, Bronx, New York 10468-1589, USA
}

(Received 24 August 2009; published 15 January 2010)

\begin{abstract}
We report evidence of the quantization of the rotational motion of solid particles containing thousands of atoms. A system of $\mathrm{CoFe}_{2} \mathrm{O}_{4}$ nanoparticles confined inside polymeric cavities has been studied. The particles have been characterized by the $\mathrm{x}$-ray diffraction, transmission electron microscopy, plasma mass spectroscopy, ferromagnetic resonance (FMR), and magnetization measurements. Magnetic and FMR data confirm the presence of particles that are free to rotate inside the cavities. Equidistant, temperatureindependent jumps in the dependence of the microwave absorption on the magnetic field have been detected. This observation is in accordance with the expectation that orbital motion splits the low-field absorption line into multiple lines.
\end{abstract}

DOI: 10.1103/PhysRevLett.104.027202

PACS numbers: 75.50.Tt, 75.45.+j, 75.75.-c, 76.30.-v

The frequency of an electromagnetic wave measured by a rotating observer is different from the frequency of a wave in the laboratory coordinate frame [1]. This is the rotational Doppler effect responsible for, e.g., the wellknown phenomenon of the phase wrap-up in the Global Positioning System (GPS). The easiest way to understand this effect is to consider a circularly polarized electromagnetic wave propagating along the axis of the rotation of the receiver, Fig. 1. The frequency of the wave, $\omega=2 \pi f$, measured at a given point in space corresponds to the angular velocity of the rotation of the electric (magnetic) field due to the wave. If the receiver is rotating mechanically at an angular velocity $\Omega$, then the frequency of the wave perceived by the receiver equals

$$
\omega^{\prime}=\omega \pm \Omega \text {. }
$$

The sign, plus or minus, depends on the helicity of the wave with respect to the direction of the rotation of the receiver. This formula has been tested by the GPS for the case of a receiving antenna making as little as 8 revolutions per second as compared to the carrier frequency of the electromagnetic waves in the $\mathrm{GHz}$ range [2].

The angular velocity can be expressed via the angular momentum $L$ and the moment of inertia $I$ as $\Omega=L / I$. The question then arises whether one can detect with the help of Eq. (1) quantization of the angular momentum of the receiver of electromagnetic waves? For a really macroscopic body this would be out of the question because of the smallness of $\hbar / I$ due to large $I$. However, for a nanoparticle the moment of inertia could be very small. A ferromagnetic nanoparticle would be a resonant receiver of the electromagnetic waves at the frequency of the ferromagnetic resonance (FMR). If such a particle is free to rotate as in Fig. 1, the FMR absorption line will be dis- placed by the angular velocity $\Omega=n \hbar / I$ with $n$ being an integer.

Most of the experiments on magnetic particles performed to date were done on systems in which particles are embedded in a nonmagnetic solid matrix. Such particles are firmly coupled with a solid and cannot rotate. Their magnetic anisotropy results in the magnetic hysteresis [3]. On the contrary, a free particle would eventually rotate towards the direction of the magnetic field as an arrow of a compass, rendering no hysteresis. Such a behavior has been previously observed in the insulating composite containing crystalline $\gamma-\mathrm{Fe}_{2} \mathrm{O}_{3}$ nanoparticles dispersed in a solid polymeric matrix [4]. Recently, we have found that a similar behavior is exhibited by $\mathrm{CoFe}_{2} \mathrm{O}_{4}$ nanoparticles in polymeric cavities. $\mathrm{CoFe}_{2} \mathrm{O}_{4}$ particles were grown by

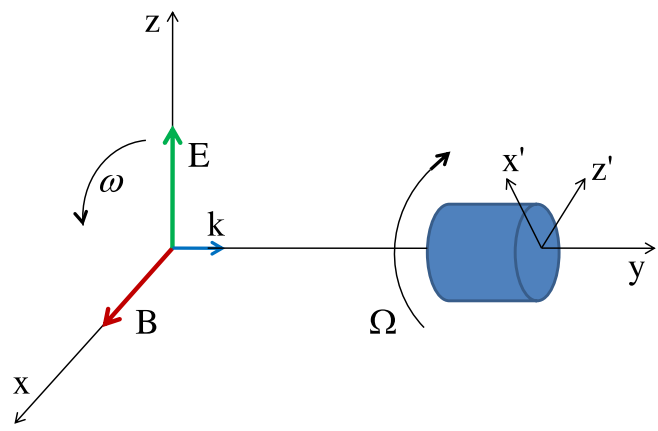

FIG. 1 (color online). Rotational Doppler effect. The frequency $\omega$ of the circularly polarized plane wave $(\omega, \mathbf{k})$ is the angular velocity of the rotation of the electric (magnetic) field due to the wave at a given point in space. The rotation of the receiver at an angular velocity $\Omega$, depending on the direction of the rotation and the helicity of the wave, adds or subtracts $\Omega$ to the frequency of the wave $\omega$, rendering $\omega^{\prime}=\omega \pm \Omega$ in the coordinate frame of the receiver. 


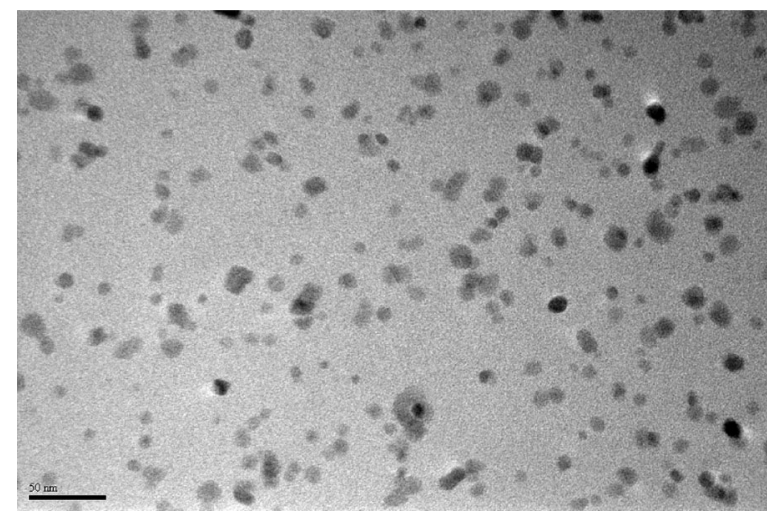

FIG. 2. TEM micrograph of $\mathrm{CoFe}_{2} \mathrm{O}_{4}$ nanoparticles in a polymer matrix.

in situ preparation [4-6]. The cross-linked polystyrene sulfonated ion exchange resin was loaded with $\mathrm{Co}(\mathrm{II})$ and $\mathrm{Fe}$ (II) salts (molar ratio 1:2) in aqueous solution, followed by thorough washings to remove the excess of physisorbed ions. The exchange resin was then exposed to an aqueous solution of $\mathrm{NaOH}$ at $60^{\circ} \mathrm{C}$, which caused the precipitation of the corresponding hydroxide that was further converted into the oxide. The presence of the nanoparticles of the $\mathrm{CoFe}_{2} \mathrm{O}_{4}$ phase was established by the $\mathrm{x}$-ray diffraction. The iron cobalt content of the samples was determined by inductively coupled plasma mass spectrometry (ICPMS). ICPMS revealed an oxide content of $12.4 \mathrm{wt}$. This agreed well with the transmission electron microscopy (TEM) images that confirmed a dilute system; see Fig. 2. The TEM with selected area electron diffraction showed that the crystallites of $\mathrm{CoFe}_{2} \mathrm{O}_{4}$ had a distribution of sizes centered at diameter $4-5 \mathrm{~nm}$; see Fig. 3. TEM microphotographs revealed mostly isometric particles but some needles were also seen. The particles did not appear to be aggregated. The presence of cavities is related to the formation of the polymer matrix. The particles grow in a successive loading and adapt to the polymer cavities. The latter prevent the formation of large particles. The largest particles of the distribution must be attached to the walls of

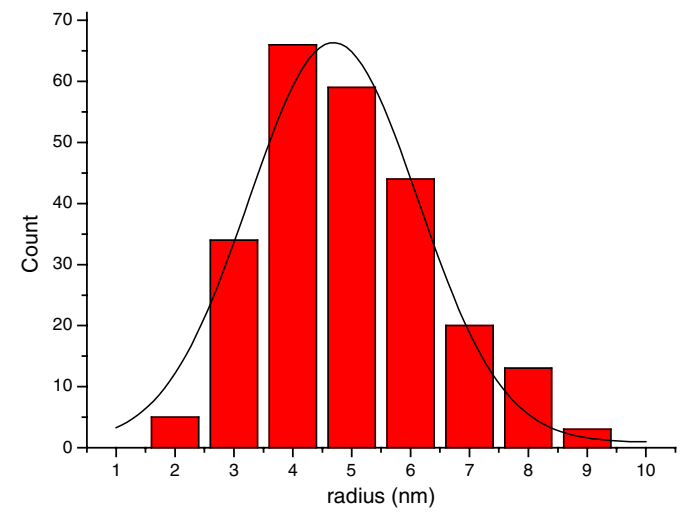

FIG. 3 (color online). Size distribution of particles shown in Fig. 2. the polymer cavities, while the majority of the smaller particles (below 2-3 $\mathrm{nm}$ in size) should be free to rotate inside the cavities. This has been confirmed by magnetic measurements; see Fig. 4. After cooling the sample in a zero magnetic field, the moments of the particles were oriented randomly such that the total magnetization, $M$, was zero. Then, a small field was applied and the temperature dependence of the magnetization was recorded. The data show that the particles that are firmly attached to the polymeric matrix are superparamagnetic above the blocking temperature of $300 \mathrm{~K}$. The magnetic moments of these particles become quickly frozen as one lowers the temperature below $300 \mathrm{~K}$. If one uses the bulk anisotropy constant of $\mathrm{CoFe}_{2} \mathrm{O}_{4}, K=2 \times 10^{6} \mathrm{~J} / \mathrm{m}^{3}$ [7], the field cooled (FC) and zero field cooled (ZFC) magnetic data are consistent with the distribution of sizes extracted from the TEM. A very steep rise of the ZFC curve at the lowest temperatures indicates the presence of a significant fraction of particles behaving as an arrow of a compass: Their magnetic moments align with the magnetic field even at a very low value of the field (below $1 \mathrm{G}$ ). We have checked that the reversal of such a very small field also reverses the magnetization of these particles. These findings have been confirmed by the ac susceptibility data recorded at $1 \mathrm{Oe}$; see inset in Fig. 4. The real part of the ac susceptibility is well described by the Curie law and is independent of the frequency, while the imaginary part of the susceptibility is zero. Note that the high-field isothermal magnetization curves are temperature independent, ruling out a significant contribution of paramagnetic ions. Large variation of the isothermal magnetization near zero field shown in Fig. 5 provides further support to the model of free particles.

The ESR measurements were performed with a commercial Bruker ESP300 spectrometer at $9.5 \mathrm{GHz}$ in a rectangular $X$-band resonant cavity generating the $\mathrm{TE}_{102}$ mode and at $34.0 \mathrm{GHz}$ in a cylindrical $Q$-band resonant cavity generating the $\mathrm{TE}_{011}$ mode. The data were taken between $-50 \mathrm{Oe}$ and $15 \mathrm{kOe}$ in the temperature range 2-300 K after cooling the sample in a zero magnetic field. At low fields, ESR data show nonresonant energy absorp-

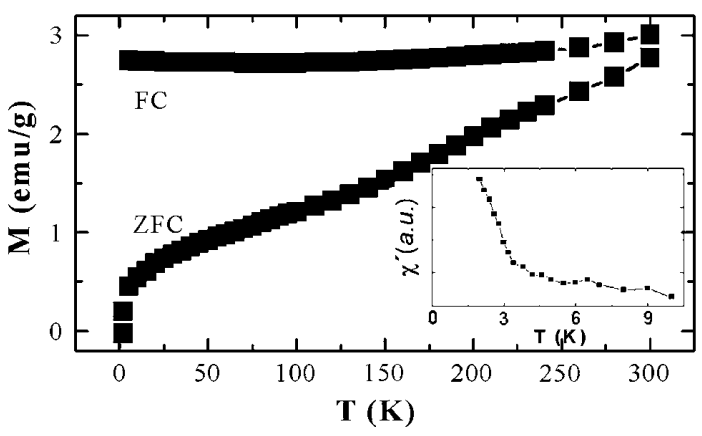

FIG. 4. Magnetization vs temperature for the FC and ZFC samples in an applied field of 25 Oe. Inset shows the ac susceptibility measured at $1 \mathrm{Oe}$. 


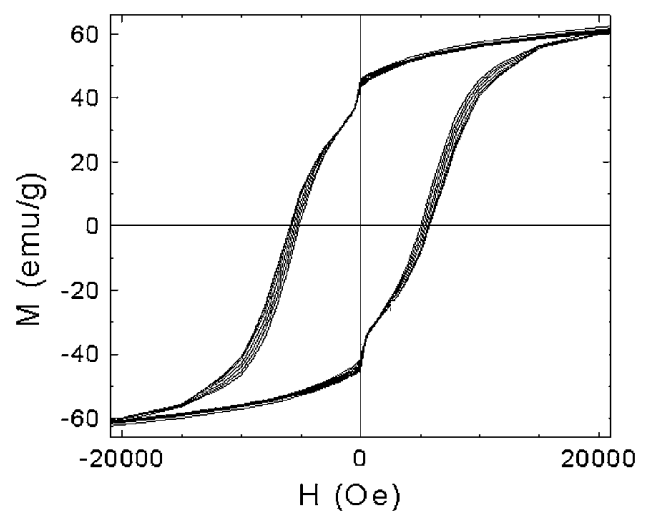

FIG. 5. Isothermal magnetization curves between $3 \mathrm{~K}$ (the outer cycle) and $50 \mathrm{~K}$ (the inner cycle).

tion at zero magnetic field at all temperatures and frequencies. It vanishes when a static magnetic field is applied. This behavior is consistent with the presence of magnetic particles that are free to rotate [8]. Such particles absorb the microwave energy by rotating together with the magnetic field of the circularly polarized electromagnetic wave. The static magnetic field suppresses this effect by maintaining a preferred orientation of the magnetic moments of the particles.

Our most remarkable observation is the stepwise field dependence of the low-field absorption of the microwave energy, Fig. 6. Various control samples have been measured to confirm that the observed feature is unique for the $\mathrm{CoFe}_{2} \mathrm{O}_{4} /$ polymer system. The curves in Fig. 6 are completely reproducible; the data have been verified in more than 100 runs. The remarkable feature of the steps is that their positions do not depend on the microwave input power or temperature. While Fig. 6 shows data taken at three different temperatures, the independence of the positions of the steps on temperature has been verified for many other temperatures between 2 and $300 \mathrm{~K}$. This rules out thermal fluctuations. In fact the steps are nearly equally spaced on the field, with a distance of $\Delta H \approx 2.5$ Oe. We have also verified that the positions of the steps and their spacing do not depend on the modulation frequency of the dc field. The strength of the low-field signal is comparable to the strength of the high-field signal due to conventional FMR of frozen particles. The data, therefore, strongly suggest that the observed steps reflect the intrinsic physics of the system.

The hysteresis in Fig. 6 is a reflection of the hysteresis in Fig. 5. If the $I(H)$ loops in Fig. 6 were smooth, they would be very similar to the data taken from numerous particulate solid systems where the anisotropy axes of the particles are frozen in random directions. The new feature of our system that was not present in particulate media studied before is the presence of free particles. It is then natural to assume that the data in Fig. 6 represent two effects: The normal smooth loop due to randomly oriented frozen particles and the absorption maxima due to free particles. The dynamics

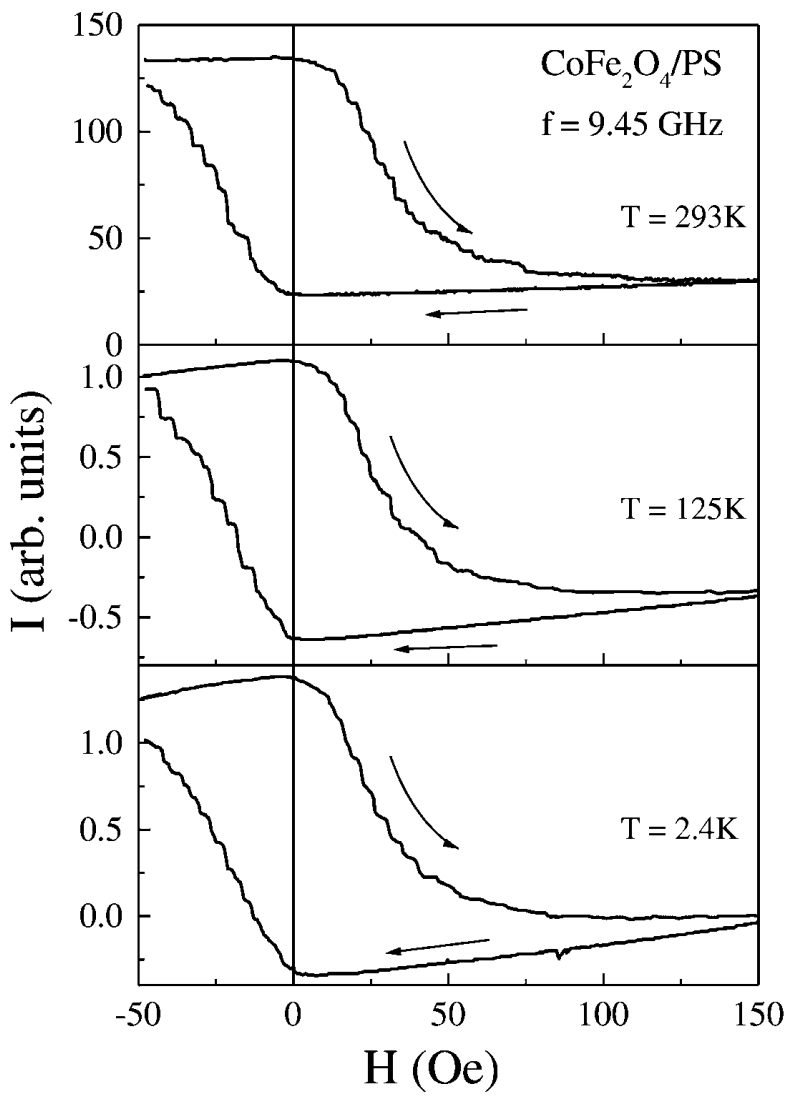

FIG. 6. Low-field ESR absorption signal at $9.45 \mathrm{GHz}$ measured at three different temperatures.

of these particles should be fundamentally different from the dynamics of frozen particles. It is also different from the dissipative dynamics of magnetic particles in ferrofluids [9]. Free magnetic clusters in beams have been studied in the past [10-12]. They exhibit a number of interesting phenomena related to the interaction between spin and mechanical degrees of freedom. Theory of mechanical rotations coupled to spins has been developed for local elastic twists in solids [13], as well as for simple oscillators like cantilevers [14], but not for threedimensional rotators.

For the sake of simplicity we shall assume (see discussion below) that the mechanical rotations that matter are the rotations of the particles about the direction of the dc field. In our experiment the direction of the field was the same as the direction of the propagation of circularly polarized microwaves. Thus the geometry of our experiment is the one depicted in Fig. 1, with the particle being the rotating receiver of the electromagnetic radiation. For a resonant absorption, the frequency of the radiation in the rotating frame of the particle, $\omega^{\prime}$, must coincide with the FMR frequency. When the magnetic moment and the anisotropy axis are aligned with the field the FMR frequency is given by [3] $\omega_{\mathrm{FMR}}=\omega_{0}+\gamma H$, where $\omega_{0}$ is the field-independent gap due to magnetic anisotropy and $\gamma=$ $2 \mu_{B} / \hbar$ is the gyromagnetic ratio for the spin. Equation (1) 
with $\Omega=n \hbar / I$ then yields

$$
\gamma H_{n}=\omega-\omega_{0}+\frac{n \hbar}{I}, \quad n=0, \pm 1, \pm 2, \ldots
$$

for the resonant values of the field. Another interpretation of this formula is the splitting of the FMR frequency,

$$
\omega_{\mathrm{FMR}}^{(n)}=\omega_{\mathrm{FMR}}-\frac{n \hbar}{I}, \quad n=0, \pm 1, \pm 2, \ldots,
$$

due to the orbital motion of the particles.

Equation (2) gives the following simple expression for the distance between the resonances:

$$
\Delta H=\frac{\hbar^{2}}{2 \mu_{B} I} .
$$

Let us estimate the size of the particle that would produce the observed value of $\Delta H$. Taking $I \sim m r^{2} \sim \rho r^{5}$ for the moment of inertia of a particle of mass $m$, mass density $\rho$, and radius $r$, one obtains from Eq. (4) $d=2 r \sim 1.7 \mathrm{~nm}$ for $\Delta H=2.5$ Oe and $\rho=5.3 \mathrm{~g} / \mathrm{cm}^{3}$ (mass density of the $\mathrm{CoFe}_{2} \mathrm{O}_{4}$ crystal). Such a diameter of free particles is consistent with the rest of our data.

The applicability of the above model requires a number of conditions. First, the distribution of the free particles over $\omega_{0}$ and $I$ should be narrowly peaked. Second, the orbital states of the free particles that are characterized by the quantum number $n$ should be occupied. The energy of the $n$th state is $E_{n}=L(L+1) /(2 I)=n(n+1) \mu_{B} \Delta H$. The condition $E_{n} \sim k_{B} T$ provides the number of the occupied states, $n_{\max } \sim\left[k_{B} T /\left(\mu_{B} \Delta H\right)\right]^{1 / 2}$. For $\Delta H \sim 2.5$ Oe, the energy $\mu_{B} \Delta H$ equals $0.17 \mathrm{mK}$. Consequently, even at our lowest experimental temperature of $2 \mathrm{~K}$, the number of occupied orbital states exceeded 100. Third, we assumed that only the rotations about the direction of the dc magnetic field contributed to the splitting of the FMR. This requires the magnetic moment $\mu$ and the anisotropy axis of the particle to be aligned with the field, which is true when $\mu H \gg k_{B} T$. Since $\mu \gg \mu_{B}$, the second and third conditions leave a broad temperature interval inside which they are fulfilled: $\mu_{B} \Delta H \ll k_{B} T \ll \mu H$. However, the size of the free particles estimated above corresponds to $\mu$ that is too low to suppress thermal fluctuations of the axis of rotation at high temperatures. In this case the particles should be treated as three-dimensional rotators. The FMR would still split into multiple lines but the exact expression may be more complicated than Eq. (2).

In conclusion, we have studied a system containing free magnetic particles in polymeric cavities. Experimental evidence has been presented that rotations of the particles split ferromagnetic resonance into multiple lines. This is the first observation of the quantization of the mechanical angular momentum in solid clusters containing thousands of atoms.

J. T. thanks ICREA ACADEMIA for financial support. The work of E. M. C. has been supported by the University of Barcelona and by the NSF Grant No. DMR-0703639.

[1] B. Mashhoon, Phys. Lett. A 306, 66 (2002); J. C. Hauck and B. Mashhoon, Ann. Phys. (Leipzig) 12, 275 (2003); B. Mashhoon, Phys. Rev. A 79, 062111 (2009).

[2] N. Ashby, Living Rev. Relativity 6, 1 (2003), http:// www.livingreviews.org/lrr-2003-1, and references therein.

[3] E. M. Chudnovsky and J. Tejada, Lectures on Magnetism (Rinton Press, Princeton, NJ, 2006).

[4] J. Tejada, X.X. Zhang, E. C. Kroll, X. Bohigas, and R. Ziolo, J. Appl. Phys. 87, 8008 (2000).

[5] R. F. Ziolo, E. P. Giannelis, B. A. Weinstein, M. P. O’Horo, B. N. Ganguly, V. Mehrotra, M. W. Russell, and D. R. Huffman, Science 257, 219 (1992).

[6] D. López, I. Cendoya, C. Mijangos, A. Juliá, R. Ziolo, and J. Tejada, Macromol. Symp. 166, 173 (2001).

[7] H. Shenker, Phys. Rev. 107, 1246 (1957).

[8] G.E. Pake and T.L. Estle, The Physical Principles of Electron Paramagnetic Resonance (W.A. Benjamin, Inc., Massachusetts, USA, 1973), 2nd ed.

[9] S. Odenbach, Magnetoviscous Effects in Ferrofluids (Springer-Verlag, Berlin, Heidelberg, 2002).

[10] D. M. Cox, D. J. Trevor, R. L. Whetten, E. A. Rohlfing, and A. Kaldor, Phys. Rev. B 32, 7290 (1985).

[11] W. A. de Heer, P. Milani, and A. Chatelain, Phys. Rev. Lett. 65, 488 (1990); I. M.L. Billas, J. A. Becker, and W. A. de Heer, Z. Phys. D 26, 325 (1993); I. M.L. Billas, J.A. Becker, A. Chatalain, and W. A. de Heer, Phys. Rev. Lett. 71, 4067 (1993); X. Xu, S. Yin, R. Moro, and W. A. de Heer, Phys. Rev. Lett. 95, 237209 (2005); S. Yin, X. Xu, R. Moro, and W. A. de Heer, Phys. Rev. B 72, 174410 (2005).

[12] J. P. Bucher, D. C. Douglass, and L. A. Bloomfield, Phys. Rev. Lett. 66, 3052 (1991); D. C. Douglass, J. P. Bucher, and L. Bloomfield, Phys. Rev. Lett. 68, 1774 (1992); D. C. Douglass, D. M. Cox, J. P. Buchwer, and L. A. Bloomfield, Phys. Rev. B 47, 12874 (1993).

[13] E. M. Chudnovsky, Phys. Rev. Lett. 92, 120405 (2004); E. M. Chudnovsky, D. A. Garanin, and R. Schilling, Phys. Rev. B 72, 094426 (2005).

[14] R. Jaafar, E. M. Chudnovsky, and D. Garanin, Phys. Rev. B 79, 104410 (2009); R. Jaafar and E. M. Chudnovsky, Phys. Rev. Lett. 102, 227202 (2009). 